Cinémas

Revue d'études cinématographiques

Journal of Film Studies

\title{
Entre l'écho des voisins et celui des cousins. Les voix croisées de la réception critique des films de Denys Arcand aux États-Unis et en France
}

\section{Denis Bachand}

Volume 7, numéro 3, printemps 1997

Cinéma québécois et États-Unis

URI : https://id.erudit.org/iderudit/1000951ar

DOI : https://doi.org/10.7202/1000951ar

Aller au sommaire du numéro

Éditeur(s)

Cinémas

ISSN

1181-6945 (imprimé)

1705-6500 (numérique)

Découvrir la revue

Citer cet article

Bachand, D. (1997). Entre l'écho des voisins et celui des cousins. Les voix croisées de la réception critique des films de Denys Arcand aux États-Unis et en France. Cinémas, 7(3), 119-136. https://doi.org/10.7202/1000951ar
Résumé de l'article

L'exploration des stratégies argumentaires déployées dans les discours critiques consacrés aux films de Denys Arcand Le Déclin de l'empire américain (1986), Jésus de Montréal (1989) et Love and Human Remains (1994) en France et aux États-Unis dévoile les filtres culturels qui régissent l'appropriation des oeuvres au sein de ces communautés interprétatives. Par ailleurs, tout en postulant que la langue et la nationalité déterminent certains traits identitaires communs aux critiques et à leurs lectorats respectifs, l'analyse permet de cerner les motifs affinitaires qui traversent et excèdent ces clivages nationaux. 


\section{Entre l'écho des voisins et celui des cousins. Les voix croisées de la réception critique des films de Denys Arcand aux États-Unis et en France}

\section{Denis Bachand}

\section{RÉSUMÉ}

L'exploration des stratégies argumentaires déployées dans les discours critiques consacrés aux films de Denys Arcand Le Déclin de l'empire américain (1986), Jésus de Montréal (1989) et Love and Human Remains (1994) en France et aux États-Unis dévoile les filtres culturels qui régissent l'appropriation des œuvres au sein de ces communautés interprétatives. Par ailleurs, tout en postulant que la langue et la nationalité déterminent certains traits identitaires communs aux critiques et à leurs lectorats respectifs, l'analyse permet de cerner les motifs affinitaires qui traversent et excèdent ces clivages nationaux.

\section{ABSTRACT}

The exploration of the strategies of argumentation used in French and American critical discourse on the films Decline of the American Empire (1986), Jesus of Montreal (1989) and Love and Human Remains (1994) reveals the cultural filters that govern the appropriation of the works in these two interpretive communities. Moreover, while postulating that language and nationality determine certain identity traits shared by the respective critics and their readers, the analysis also brings to light the motifs of affinity that crisscross and overcome these national divisions. 
Le cinéma québécois a connu un retentissement international sans précédent lors de la sortie du Déclin de l'empire américain de Denys Arcand en 1986. Aucun film québécois réalisé en version originale française n'avait suscité jusqu'alors autant d'échos favorables dans le monde ni en particulier aux États-Unis, marché réputé singulièrement imperméable aux productions étrangères. Fort de cette renommée, et bénéficiant conséquemment d'un appareil promotionnel de diffusion plus performant, le cinéaste allait rééditer l'exploit avec Jésus de Montréal en 1989'. Étions-nous en présence d'une vague de succès qui allait se confirmer et s'amplifier ? Arcand saurait-il exploiter une veine propice à la poursuite de sa conquête du monde et des États-Unis ? Plusieurs le croyaient et le souhaitaient au moment de la sortie de Love and Human Remains en 1994. Les conditions semblaient en effet réunies pour assurer un accueil convaincant: Arcand venait de tourner son premier film en langue anglaise, certains des comédiens étaient connus du public états-unien ${ }^{2}$, et les problématiques de la "génération X" étaient d'actualité. Malheureusement, l'engouement ne se confirma point et le film, malgré les coupes effectuées par le cinéaste à la suite de l'attribution d'une première cote restrictive en interdisant l'accès au public cible des adolescents ("NT-17»), attira moins de spectateurs que les deux précédentes productions ${ }^{3}$. Cette aventure récente témoigne des aléas de la réception de l'œuvre d'art, qui demeure un pari inédit, joué et répété au cas par cas.

Cette étude porte sur l'accueil que la critique a réservé à ces films aux États-Unis et en France, où ils ont généré une substantielle couverture médiatique, à l'exception toutefois notable (et éloquente) de Love and Human Remains qui n'a pas encore été distribué dans le réseau commercial français ${ }^{4}$. Plus spécifiquement, et à travers l'exploration des stratégies argumentaires déployées par le discours critique, l'analyse cherche à dévoiler les mécanismes qui régissent l'appropriation des œuvres au sein d'une communauté interprétative donnée. Par ailleurs, tout en postulant que la langue et la nationalité déterminent certains traits identitaires communs aux critiques et à leurs lectorats respectifs, la comparaison vise à cerner les motifs affinitaires qui traversent et excèdent ces clivages territoriaux. De plus, la mise 
en relief et les rapprochements des filtres culturels propres à ces différents contextes de réception nous invitent à questionner l'européanité et l'américanité de l'identité québécoise, souvent prises pour acquises, à quoi font écho les commentateurs étrangers.

\section{L'écran de la langue}

D'entrée de jeu, abordons la question pour mieux nous en délester: la langue des films differe. Ce constat, qui s'impose d'emblée comme une évidence banale, entraîne pourtant à sa suite son lot de considérations. En effet, les Français voient, mais surtout entendent les films en provenance de " [...] ce fragment bizarre d'Amérique" dans leur langue maternelle ou plutôt dans une variante idiomatique comportant " [...] d'autres anglicismes que [les leurs]", et de laquelle émane "[...] une franchise un peu rustaude qui est une vertu proprement québécoise" (Léonardini). Taxé par les mouvements de l'histoire, dont il conserve les empreintes de son élaboration, le rapport à l'autre révèle ainsi une part du faisceau connotatif sur lequel se fonde les horizons d'attente. La majorité des spectateurs français anticipent et recherchent ces traits convoyeurs de couleur locale au point où leur absence fera douter de l'authenticité de certains films qui les exhibent trop peu ou pas du tout (d'autant plus lorsqu'il s'agit d'un film en anglais comme Love and Human Remains projeté au Festival de Blois en 1994). De fait, les spectateurs entretiennent une relation ambiguë avec la langue québécoise: bien que les variantes linguistiques engendrent généralement des prédispositions favorables, lorsque l'épaisseur du signifiant se fait trop opaque, les difficultés de compréhension refrènent l'engouement et contribuent à détourner la fiction vers le documentaire (Bachand, 1996). La référence linguistique constitue un relais fossilisé, conducteur et inducteur de perceptions et d'émotions. Aussi la critique française, sauf en de rares exceptions, emprunte le parcours obligé de son rappel pour caractériser les films dont elle traite, au risque parfois d'en amplifier l'importance et la portée comme c'est le cas pour ce chroniqueur (M.-N. T.) du Figaro magazine qui, soucieux d'effet, impose un gigantesque et grotesque «Tabernacle!» (T, M.-N., 
Figaro magazine) en sous-titre à sa critique de Jésus de Montréal. Même le relais du français international ne parvient pas toujours à déjouer ce recours aux formules stéréotypées de l'échange interculturel: "Le plus amusant dans ce film [Le Déclin de l'empire américain], c'est l'accent québécois avec lequel les acteurs disent leurs cochonneries" (Anonyme, France soir).

Rien de tel évidemment chez nos voisins du sud qui sont exposés, eux, à des versions doublées ou encore en langue originale anglaise, comme Love and Human Remains qui laisse poindre à peine quelques traits locaux: "Only few casual Canadianisms and the general neatness of the bleak urban surroundings reveal that the setting is no U. S. metropolis" (Campbell). La traduction et l'anglais canadien instillent ainsi des effets de familiarité avec les univers construits par la fiction filmique. Alors que la parenté linguistique provoque un régime de différenciation (ah! ces Québécois ou Canadiens), la traduction véhicule le familier, le connu, le même par quoi est assimilé et simultanément oblitéré la spécificité de la culture d'origine. La filiation génère des effets spéculaires trompeurs là où l'étrangeté ouvre des brèches à la transparence. L'autre est ainsi aisément assimilable au nous: "[...] Canadians are like Americans, only less so [...]" (Cheshire), formule lapidaire qui révèle éloquemment le procédé de concaténation par lequel l'instance d'énonciation compte soumettre les propositions inédites à son système référentiel.

Bien que la critique états-unienne rappelle systématiquement l'origine French Canadian de Denys Arcand, elle ne manque pas, faisant l'économie de la distinction québécoise, d'attribuer à l'œuvre la froideur générale que l'on associe généralement aux films canadiens dans leur ensemble. L'argumentation fonctionne par télescopage métaphorique par quoi s'opère une condensation réductrice du champ symbolique. Prenant conativement à témoin son destinataire, suggérant ainsi le partage d'un répertoire commun relatif au Canada, le discours associe les configurations géographiques et climatiques au soi-disant "caractère national " et en fait découler les choix esthétiques. Cette vision stéréotypée pousse loin ses racines dans les représentations mythiques proposées du Canada par des films comme North- 
west, Big Snows ou Great Woods tournés à Hollywood au début du siècle (Berton, 1975). Aussi, le cliché de la nordicité peut-il inspirer des propos du type "Canadian films, as we know are weird, but in a comfortingly predictable way. With an emotional temperature just this side of a snowball's, they tend to be cerebral and coolly distanced, albeit more by reflex than choice " (Cheshire). Ce type de raccourci conforte le public dans ses certitudes douteuses et contribue à la cristallisation des structures d'accueil.

La migration d'un univers linguistique à un autre joue un rôle capital dans l'économie générale des échanges interculturels. Elle force l'interrogation sur le passage, ou l'altération, des traits spécifiques de l'altérité soumise à l'épreuve des équivalences. Cela revêt une importance particulière lorsque le style d'un cinéaste se caractérise par un usage abondant de dialogues: "A typical Arcand film meanders purposefully through shrewd, mordant conversations among friends" (James). Que ces conversations se déroulent en français ou en anglais importe cependant peu dans la mesure où l'esprit des cultures d'arrivée est respecté par le génie propre de chacune des langues. Comme l'affirme un critique se référant à plusieurs cinéastes qui ont choisi de s'exprimer dans un autre idiome que leur langue maternelle: "What's more the directors haven't been handicapped by the artistic equivalent of driving on the other side of the road. They are retaining their distinctive movie diction even as they work with a new lexicon" (Iorn). Parions par ailleurs que la plupart des spectateurs ne remarquent pas spontanément qu'ils sont en présence d'une version doublée du Déclin de l'empire américain ou de Jésus de Montréal, et qu'ils seraient les premiers surpris d'apprendre que Love and Human Remains était le premier film que Denys Arcand réalisait en langue anglaise.

\section{Le Déclin de l'empire américain}

Genre, scénario, choix des interprètes et du titre font partie des ingrédients sur lesquels tablent généralement les producteurs pour assurer le succès d'un film. Bien qu'il n'existe pas de recette absolue en matière de sujet, son adéquation aux enjeux actuels d'une société représente un atout non négligeable. Effet de 
hasard plus que de calcul concerté, encore que l'observation attentive de l'actualité puisse fournir des indications quant aux questions qui hantent l'imaginaire collectif, le choix d'une thématique se révèle déterminant dans l'attrait qu'un film peut engendrer auprès du public.

Il n'est pas indifférent par exemple que la morosité, le défaitisme et les difficultés relationnelles qui trouvent un lieu d'expression dans Le Déclin de l'empire américain aient rallié une frange importante du public du milieu des années quatre-vingt. En effet, une grande part de l'interpellation provoquée par le film ressortissait à la nature des questions irrésolues d'une génération blasée en quête d'un sens à donner à ses actions. En prime, les sujets proposés s'offraient sous une forme humoristique propice à une évacuation symbolique de type cathartique génératrice de plaisir. Les critiques états-unienne et française ont abondamment encensé le film en soulignant la lucidité, la perspicacité et la maîtrise de son auteur (Bachand, 1995 et Pérusse, 1992). Connu depuis les années soixante-dix en France, où $L a$ Maudite Galette (1971), Réjeanne Padovani (1973) et Gina (1974) lui ont permis d'amasser un capital de sympathie auprès de la critique, Arcand se voyait maintenant hissé au sommet de la renommée. Appuyant et relayant les jugements de la critique française, les commentateurs américains y décèleront la griffe d'un "[...] sardonic, sharp-eye chronicler" (James) qui place la réflexion au faîte de ses préoccupations esthétiques: " [...] thought is the hallmark of Arcand films" (Julian). La critique américaine situera Le Déclin de l'empire américain dans la filiation des films européens d'art et d'essai et rappellera sa contrepartie française en comparant la facture aux œuvres d'Éric Rohmer et de Woody Allen, tout en le rapprochant du Big Chill de Laurence Kasdan (1983), plus familier de ses lecteurs.

Bien que les publics soient composites et que les sujets traités déclenchent des résonances variables d'un spectateur à un autre, des convergences se dégagent néanmoins qui découlent du modelage des mentalités au sein d'institutions communes. Aussi, l'onde de choc des propositions filmiques se répercute-t-elle le long d'un tracé qui prend sa source dans le substratum fondateur des systèmes de valeur dont la croyance religieuse constitue l'un 
des vecteurs privilégiés. S'explique ainsi que Le Déclin de l'empire américain et Jésus de Montréal aient connu des fortunes inversement proportionnelles dans des pays aux traditions religieuses différentes. Le premier a été mieux accueilli dans les pays catholiques comme la France, alors que le second a davantage correspondu aux goûts des publics de tradition protestante comme aux États-Unis's. Il est instructif à cet égard d'observer que les Américains ont généralement ressenti Jésus de Montréal comme une réponse progressiste, un repentir faisant suite à la "confession publique" du Déclin de l'empire américain, alors que les Français auraient plutôt tendance à percevoir l'allégorie religieuse comme expression d'une régression moralisatrice de "l'abbé Arcand " qui nous propose "l'épître d'un pitre" (S. G., L'Express).

\section{Jésus de Montréal}

Bénéficiant de la réputation acquise avec Le Déclin de l'empire américain et profitant de l'effet d'entraînement provoqué par le film de Scorsese, The Last Temptation of Christ (1989), auquel on compare avantageusement Jésus de Montréal, Arcand est louangé pour sa mise en scène et sa direction d'acteurs. On souligne par ailleurs son ingéniosité scénaristique en opposition au traitement réducteur, malhabile et violent qu'a offert Scorsese de l'œuvre de Kansansakis :

Il y a peu de chances que Jésus de Montréal soulève un scandale semblable à celui du film de Scorsese. $\mathrm{La}$ Dernière Tentation... était un film presque naïf, dans sa volonté de représentation du Christ. Alors que Jésus de Montréal accumule les roublardises - au bon sens du terme (Ferenczi).

Arcand's residual Catholicism doesn't overpower Jesus of Montreal. For all his pushiness with parallels, he is understated compared to Scorsese in The Last Temptation. [...] Jesus of Montreal succeeds better at contemporizing the Gospel because, whatever its faults, it doesn't posture as much, as heavily - or as neurotically (O’Brien).

Ruse et finesse définissent ainsi une approche stylistique qui reçoit l'aval d'une critique fortement sollicitée par la polémique 


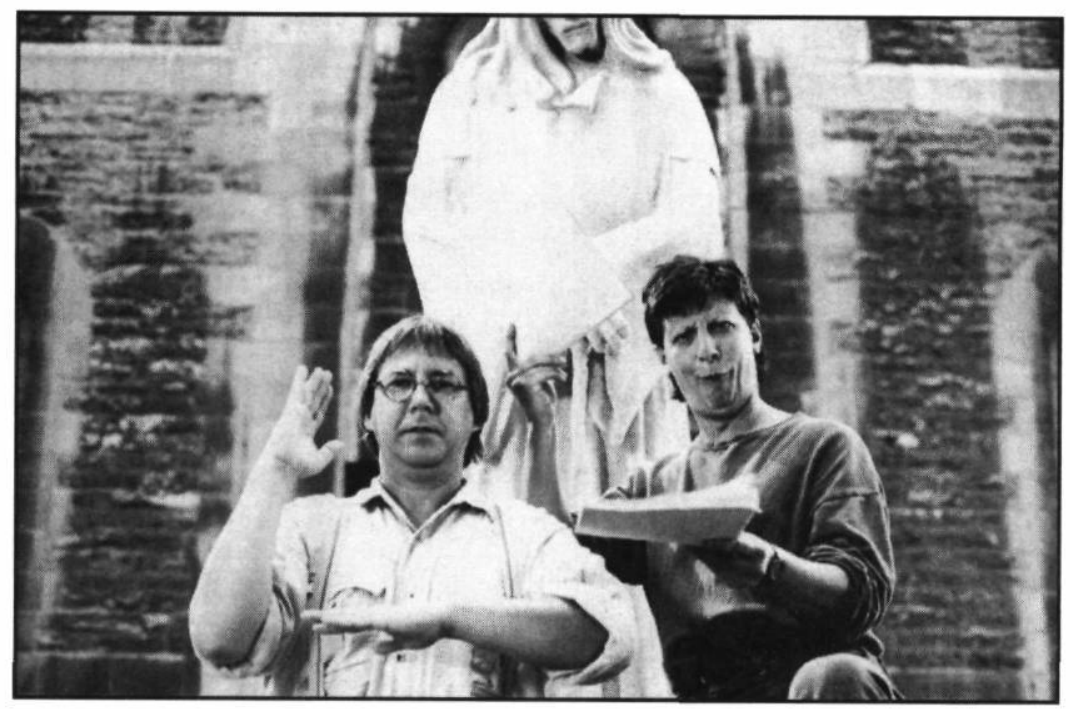

Jésus de Montréal de Denys Arcand (1989)

Collection Cinémathèque québécoise

entourant le film de Scorsese. La mise en abyme arcandienne est en effet perçue comme une distanciation propice à endiguer un questionnement potentiellement blasphématoire. En d'autres mots, l'imitation de Jésus-Christ à laquelle se livrent les comédiens montréalais est favorablement ressentie parce qu'elle résiste à la substitution littérale. De fait, le film réussit exactement là où celui de Scorsese irrite, c'est à dire dans le registre de la sexualité, qui est affichée brutalement à l'écran dans The Last Temptation, alors qu'elle est subtilement évoquée dans le film québécois: "Arcand's Christ and Magdelene are shown as intimate, and loving, but the closest we get to sexual contact is a short scene of Wilkening washing Bluteau's upper body in a bathtub. Could Scorsese have been similarly indirect ? Of course" (O’Brien).

On aurait vraisemblablement préféré que le cinéaste américain eût procédé de la sorte, établissant une mise à distance moins compromettante: "The less heavy-handed Jesus of Montreal conveys by sweet suggestion what The Last Temptation so dumly overplayed" (O’Brien). Comble d'ironie, Scorsese se voit accusé de ce qu'il comptait dénoncer: les fondements puritains 
de l'obsession sexuelle de l'époque moderne — "The sex-made Puritanism he ostensibly wanted to attack" (O’Brien). De fait, les deux perspectives sont diamétralement opposées: alors que le film québécois insiste sur l'aspiration à la divinité d'un homme, le texte de Kansansakis ramène la divinité aux dimensions humaines jusqu'à la réduire à la fantasmatique sexuelle qui a scandalisé les fidèles et déclenché les foudres de l'Église lors de la sortie de son adaptation cinématographique. La transposition distanciatrice d'Arcand, "[the] fragmented directoral style" (Kreps), «[...] toute en rupture de ton" (Macia) qui «[...] brise l'émotion" (Murat) en jouant alternativement des codes de la comédie et de la tragédie, installe plutôt une dialectique qui interdit l'adhésion au premier degré alors que la reconstitution historique "[...] filmée du point de vue de Dieu" (J.-M. F., Le Point) de Scorsese indispose et provoque l'irritation en forçant l'adéquation.

Arcand n'épargne rien ni personne. Pas même son film. Éprouve-t-on une vraie émotion en voyant ses comédiens jouer la Passion? Voilà Arcand, tout affolé à cette idée, qui brise l'émotion, qui la détruit sciemment, au moyen d'un guide aux interventions bouffonnes: "Fin du troisième tableau, m'sieurs-dames. Et pour le quatrième, attention aux marches!» (Murat).

Ce recadrage qui produit des raccords inattendus entre le champ et le hors-champ libère l'entrave de la fascination spectatoriale en dirigeant l'attention dans l'ordre du discursif qui se saisit du cinéma comme référent. Arcand sait jouer du code...

La structure narrative, construite selon le principe de l'alternance des régimes de l'allégorie, et qui propose une relecture de la tentation du Christ relatée dans les évangiles, est accueillie différemment selon les allégeances religieuses et les libertés que l'on s'accorde avec le dogme. Cette nouvelle herméneutique appliquée à la critique des médias est généralement perçue comme une réhabilitation du message originel: "[...] the kind that both Puritans and non-Puritans are likely to miss" (O'Brien). La référence à la tradition puritaine fondatrice de la morale états-unienne n'est évidemment pas innocente, car elle s'inscrit au cœur de la dynamique axiologique qui préside à 
l'évaluation des œuvres (Dôle, 1996). La convoquer ici témoigne d'un ancrage profond de l'imaginaire que le critique exhume pour rallier son lectorat autour de valeurs reconnues, qu'elles soient partagées ou pas importe alors moins que le fait qu'elles agissent comme repères à la communication. Ne reste plus qu'à la controverse de s'ériger sur la délimitation des aires de dissonance potentielle suscitée par le code moral en vigueur. Des voix discordantes jugeront sévèrement la désinvolture avec laquelle le cinéaste québécois traite d'un sujet aussi grave: "The theological contrasts between the church, as represented by Father Leclerc, and the modern-day prophet, Daniel, are too simplistic to have any significance" (Garcia); et son style satirique n'est pas prisé de tous même si on lui reconnaît certains talents pour la comédie:

Arcand is a fine technician, with a very special talent for comedy. [...] But when the director is attempting satire, he fails. His understanding of the material - in this case the meaning of The Passion and its potential for social commentary - is so superficial that much of the script is filled with one-liners on the virtues of drinking a "Virgin Mary" and eating "Lobster Magdalene». [...] Form is more powerful than content in this film. [...] We are looking at the cross and seeing just the fine hew of the wood (Garcia).

La critique reconnaît qu'il n'est pas le cinéaste du divertissement léger et que chacun de ses films porte la marque d'un travail intellectuel appréciable: "Few directors are as consistently intelligent and provocative as Denys Arcand [...]" (Julian) remarque un critique américain donnant le change à un homologue français qui estime que le cinéaste " [...] fait preuve d'une grande intelligence et de beaucoup de talent. Il ne démontre pas, ne sermonne pas, ne tient pas sa parallèle trop serrée entre Daniel et Jésus" (Braudeau). Appréciation que ne partage cependant pas l'ensemble de sa profession, alors que le journaliste de Libération, particulièrement caustique, friand de jeux de mots et nourri d'anticléricalisme républicain, affuble Arcand "l'archange» (qui, à l'exemple de l'Albatros de Baudelaire s'écorche les ailes au passage) du titre "[...] d'abbé [qui] culpabilise 
son spectateur" en proposant un "[...] film balourd dans la forme, prêchi-prêcha dans le fond et nettement baba-cool sur les bords $[. .$.$] " (Anonyme, Première).$

En dépit des mérites qu'on lui accorde, Arcand, tout comme Scorsese, prête flanc au retournement critique de ses thématiques, ainsi on lui reprochera "[...] que son film [s'inscrit] dans ce qu'il prétend dénoncer: une exploitation médiatique, elle aussi, d'un sujet à la mode" (J. M., Cahiers du cinéma). Point de vue motivé peut-être par un raccourci de facilité journalistique, mais qui illustre bien la difficulté épistémologique de produire une critique dans les termes du code que l'on remet en question (l'œil est condamné à ne pouvoir s'observer que dans le miroir). L'exorcisme des démons de la collusion avec le show biz (les marchands du temple) produit de l'art, et de la bonne conscience. En somme, Arcand utiliserait des formes et des formules qui permettent à tout un chacun de se reconnaître, à commencer par lui-même...

Ce brouillage constant des pistes, ce parasitage permanent d'un aspect du sujet [la relecture du texte évangélique] par un autre [la critique des médias] permet, à l'image de ce qui se passait dans Le Déclin de l'empire américain, de satisfaire les plus opposés dans un enthousiasme parfaitement consensuel (J. M., Cahiers $d u$ cinéma).

\section{Love and Human Remains}

La jeunesse occupe une niche privilégiée dans l'imaginaire de Denys Arcand. Elle constitue de fait un vecteur majeur dont les contours se précisent et s'accentuent de film en film. La récurrence de cette figure rappelle la dynamique d'un mythe personnel à l'œuvre au cœur de la pulsion créatrice. Cette préoccupation revêt toute sa dimension et sa densité avec Love and Human Remains (1994), que le cinéaste québécois adapte de la scène en s'associant son auteur Brad Fraser comme scénariste. La parenté des thèmes est évidente et on comprend pourquoi Arcand, séduit par la pièce, a décidé pour la première fois de porter l'œuvre d'un autre à l'écran ${ }^{6}$. Certains n'hésitèrent d'ailleurs pas à suggérer que les personnages de Fraser pourraient être les 


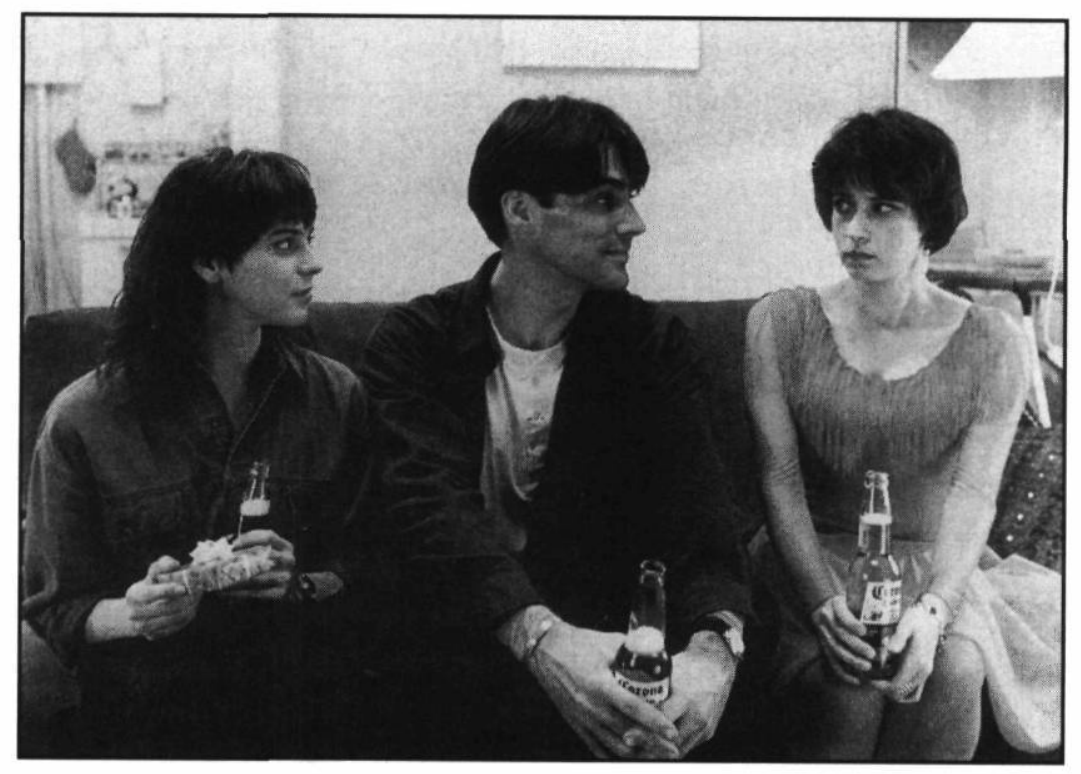

\section{Love and Human Remains de Denys Arcand (1994)}

Collection Cinémathèque québécoise

enfants de ceux du Déclin de l'empire américain ${ }^{7}$. Ces dignes progénitures des petits-bourgeois baby boomers luttent avec l'énergie du désespoir prêtée aux jeunes de la "génération X", celle des laissés-pour-compte qui ne trouvant pas leur place dans la société de consommation héritée de leurs aînés vivent d'expédients "en attendant "... Ce désœuvrement les amène à la recherche éperdue de l'amour, de l'amitié ou plus simplement de contacts humains peu importe d'ailleurs le sexe des partenaires, car l'indécision sexuelle participe de l'image stéréotypée d'une génération vouée à l'échec de sa quête identitaire.

Le film a été généralement bien accueilli dans les milieux homosexuels (presse et festivals) où l'on a souligné la justesse de la représentation qui y était offerte des gais et lesbiennes. Les comparaisons se font fréquentes et l'adaptation est jugée plus subtile que la pièce sans que le sens original ne soit édulcoré par le resserrement cinématographique du propos: délaissant le confinement des rôles clichés de marginaux, les personnages revêtent des identités qui ne sont pas réductibles à leur pratique 
sexuelle. Ils sont perçus comme des êtres entiers qui ont des vies "normales" même si l'on peut s'étonner que cela étonne, comme le laisse sous-entendre le commentaire suivant: "One of the amusing ironies of Love and Human Remains, is that the "straight" people in the film are far more sick and twisted than the gay ones" (Verniere), comme s'il était entendu que les gais et lesbiennes soient des dépravés, aussi le film opère-t-il en quelque sorte une salutaire réhabilitation. Mais le grand public, lui, a boudé le film et la critique l'a généralement pourfendu: "[...] uninspired. An emotional showdown" (Neman); "More often this Canadian film is just unpleasant" (Horton); "Does Arcand think a movie about alienation should alienate the audience ?" (Rickey).

Les filiations thématiques ne suffirent apparemment pas à reproduire le sentiment d'urgence attribué aux œuvres d'auteur du cinéaste: "One wonders what he was doing making this movie in the first place" (Neman). Le malaise se concentre particulièrement sur la forme imprimée à la narration par un montage ostentatoire fait de ruptures déroutantes et de raccords abrupts où l'on passe indistinctement d'un lieu à un autre, un peu à l'exemple de cet omniprésent zapping qui opère un collage télescopique des images décousues provenant d'un peu partout à travers le monde: "[...] the disparate parts fail to create enough provocative dissonance. Moreover, the movie seems a little too pleased with its own cleverness" (Denerstein). La construction bipolaire typique de l'écriture dialectique d'Arcand dégénère en cauchemar où faseye le sens dans l'éclatement des parcours labyrinthiques :

Unable to manage one mood, it tries for two; rather than weaving both ideas together, it cuts between them. So we get a funny dating scene, and then a hand-held-camera stalking. Kisses, then torture. Warm soft sheets, and then an earring ripping from a earlobe. It's enough to give you whiplash (Whitty).

L'usage de poncifs de raccords: appels téléphoniques, arrivées abruptes de personnages dans la pièce, sans parler du truc éculé des prédictions d'une voyante accentuent l'inconfort généré par les rapprochements qui se font de plus en plus insistants avec 
l'esthétique des romans-savons (sit com et soap opera) de la télévision américaine. Le feuilleté narratif s'aplatit ainsi paradoxalement dans ce qui devient une parataxe baroque, touffue et incohérente: "It's Nightmare on Melrose Place" (Verniere). "That's an overdose of ideas [...] Imagint TV's Friends as directed by Pedro Almadovar" (Denerstein). "What seemed terminally narcissistic onstage here seems an amusingly jagged soap opera $[\ldots]$ " (Car).

L'intertexte télévisuel est convoqué à plusieurs niveaux. D'abord par les protagonistes qui en font un leitmotiv existentiel :

- Candy: Je veux plus que du sexe.

— Lover : Dieu a inventé la télévision pour ça...

Puis par l'intermédiaire du personnage de David, qui est une ex-star d'une série télévisée, interprété par Gibson, lui-même identifié aux séries Tales of the City (PBS) et Chicago Hope $(\mathrm{CBS})^{8}$. Les fictions répercutent leurs échos croisés. L'usage abondant de gros plans et la succession de rencontres susceptibles d'installer des continuités entre les personnages inscrivent par ailleurs des parentés formelles qui contribuent à l'hybridité esthétique prêtée au film: "It's as if Fatal Attraction had bumped into the hit television series Friends" (James), mais le comedythriller ne parvient pas au même niveau de conviction. Il s'affaisse après quelques minutes et la finale est perçue comme un revirement invraisemblable, comme si le film ne parvenait pas à boucler les avenues ouvertes par le dispositif narratif qui contrevient à la stratégie du happy end: "There is an uplifting, unconvincing twist at the end [...]" (James).

Les liaisons intertextuelles fonctionnent également dans leur rapport à d'autres cinéastes connus du public états-unien, et en particulier en rapport à Atom Egoyan, que la présence de son interprète fétiche Mia Krishner impose d'emblée. On voit ainsi à l'œuvre un processus d'édification d'une représentation forcément réductrice des caractères nationaux, véhiculée par les codes rhétoriques propres au genre journalistique contraint aux raccourcis et aux formules chocs: "With Atom Egoyan's Torontoset Exotica and Arcand's Montreal-based Love and Human 
Remains Canada is beginning to look like the nation founded by Master and Johnson" (Verniere).

\section{À la croisée des voies}

Si l'exploration des discours critiques permet d'isoler certains traits spécifiques qui lient la réception des œuvres au moment du tamisage par les filtres culturels propres à un contexte national particulier, elle met également en évidence les tracés de convergence qui les irriguent. Ces derniers s'expliquent, pour une part, par la circulation des informations relayées au sein de la classe critique et, d'autre part, par le partage de valeurs communes aux pays industrialisés de l'hémisphère Nord. Citoyenneté, langue et culture n'étant pas réductibles l'une par l'autre, l'analyse doit se garder de la tentation de construire des homogénéités factices. Aussi les réfractions spéculaires de soi à l'autre, constitutives du processus identitaire, renvoient-elles à la polyphonie des relais discursifs qui alimentent les compétences culturelles que chaque critique actualise dans sa pratique de communication.

Les contextes de réception sont perméables et ils entretiennent des liens de légitimation réciproque. La critique états-unienne s'appuiera par exemple sur les commentaires de sa contrepartie française à l'occasion du Festival de Cannes pour faire l'éloge du Déclin de l'empire américain ou encore établira la crédibilité du cinéaste en rappelant les succès de ses films au Québec et en France. Par ailleurs, les filiations linguistiques qui affectent le rapport du public français aux films québécois n'interviennent évidemment pas aux États-Unis, où c'est plutôt le filtre de l'européanité qui colore la saisie des œuvres. Plus réceptifs à l'aura de l'auteur et à l'image générale de la cinématographie québécoise, la critique française quant à elle recourra fréquemment au concept de l'américanité pour se représenter les réalités appréhendées par l'intermédiaire des films. On a vu également comment la morale érigée sur les fondements religieux pouvait contribuer à spécifier les codes de réception en vigueur dans chacune de ces sociétés. Par ailleurs le cas de Love and Human Remains pose le problème particulier de l'adaptation et de la cohérence narrative. Si le scénariste n'a pas complètement réussi la transposition de sa pièce, le réalisateur n'a pas su imprimer le style qu'on lui 
connaissait. Cela pourrait expliquer en partie l'absence du film des écrans français.

Denys Arcand, comme tout créateur, est le produit de l'ensemble des différents relais institutionnels qui ont imprégné sa formation personnelle d'abord, puis de cinéaste ensuite. Né dans une société fortement religieuse et puritaine, éduqué aux valeurs françaises classiques dans un contexte nord-américain, il a été appelé à résoudre ce chassé-croisé d'influences. La réception de ses films à l'étranger témoigne qu'il a su opérer une synthèse originale en créant une œuvre aux résonances locales et aux échos internationaux.

\section{Université d'Ottawa}

\section{NOTES}

1 Le film s'est maintenu 24 semaines au "Top 50 " comparativement à 18 pour Le Déclin de l'empire américain. Jésus de Montréal récolta 1,6 millions \$ durant sa présence sur la liste de Variety alors que le précédent avait récolté 1,1 million \$. Sans rééditer l'exploit inédit du Déclin de l'empire américain, le film connut beaucoup de succès au Canada où il remporta 12 Génies de même que sur la scène internationale: Jésus de Montréal participa à la course aux Oscars dans la catégorie des meilleurs films étrangers après avoir remporté le prix du jury et le prix œcuménique au Festival de Cannes en 1989.

2 Mia Kishner, qui interprète le rôle de la prostituée voyante, est connue pour son rôle d'étudiante dans Exotica alors que Gibson, qui interprète le rôle principal de David, a joué dans plusieurs films et séries télévisées: Far and Away, The Age of Innocence, Barcelona, Sleep With Me, Chicago Hope (CBS) et Tales of the City (PBS).

3 Le film récolta seulement $850000 \$$ selon les données recueillies auprès du producteur Trafic.

4 On dénombre respectivement 48 et 46 articles consacrés au Déclin de l'empire américain et à Jésus de Montréal en France comparativement à 58 et 28 aux ÉtatsUnis. Voir les répertoires analytiques du Centre de recherche cinéma/réception à l'Université de Montréal. Présenté en première mondiale au Festival de Toronto, le film n'a pas été retenu pour la compétition au Festival de Cannes, soi-disant parce qu'il serait davantage " [...] un film nord-américain que français" selon son producteur Roger Frappier (Le Devoir, 13 septembre 1993, p. B8).

5 Entrevue de Denys Arcand réalisée par Michel Dolbec (La Presse, 8 mars 1994).

6 Le titre original de la pièce de Brad Fraser est Unidentified Human Remains and the True Nature of Love.

7 Voir, par exemple, les propos rapportés à l'occasion de l'avant-première dans $L a$ Presse du 17 février 1994, p. D8.

8 Mais pas tout à fait une vraie star...! «Oh, and did I mention that David used to be a TV star ? Not a real TV star, in the sense that he was on American TV, but enough of a Canadian TV star that he wasn't waiting for a living" (Mike LaSalle, "Loves Rules a Snapier Human Remains ", SF Chronicle, 23 juin 1995, p. C3). 


\section{OUVRAGES CITÉS}

Bachand, Denis. "La réception critique du film Le Déclin de l'empire américain aux États-Unis ". Quebec Studies, nº 19 (1995), p. 155-168.

Bachand, Denis. «Figures de spectateurs. Étude exploratoire du public du Festival du cinéma québécois de Blois", dans Michel Larouche (direction), L'Aventure du cinéma québécois en France. Montréal : XYZ éditeur (1996), p. 85-106.

Berton, Pierre, Hollywood's Canada. The Americanization of Our National Image. Toronto: McClelland and Stewart Limited, 1975.

Dôle, Robert. Le Cauchemar américain. Essai sur les vestiges du puritanisme dans la mentalité américaine actuelle. Montréal: VLB éditeur, 1996.

Pérusse, Denise. "Réception critique et contexte: à propos du Déclin de l'empire américain ". Cinémas, vol. 2, n 2-3 (1992), p. 89-105.

\section{CORPUS CRITIQUE CITÉ}

\section{FRANCE}

Anonyme, "Le déclin de l'empire américain. Ils ne parlent que de ça ", France soir, 4 février 1987.

Anonyme, "Jésus de Montréal", Première, n 147, juin 1989, p. 28.

Braudeau, Michel. "Rédemptions ", Le Monde, 17 mai 1989.

F., J.-M. " Denys Arcand: spirituel ", Le Point, 15 mai 1989.

Ferenczi, Aurélien. "Sélection officielle/en compétition. Jésus de Montréal de Denys Arcand ", Le Quotidien de Paris, 15 mai 1989.

G., S. «Denys Arcand monte au ciel ", L'Express, 19 mai 1989.

Léonardini, Jean-Pierre. "Pas d'œil sec au Québec", L'Humanité, 16 mai 1989.

M., J. "Jésus de Montréal ". Cahiers du cinéma, n 421, juin 1989, p. 52.

Macia, Jean-Luc. "Portrait du Christ en acteur canadien ", La Croix, 17 mai 1989.

Murat, Pierre et Claude-Marie Tremois. "Jésus de Montréal", Télérama, n²053, 17 mai 1989 , p.52-53.

T., M.-N. "Jésus de Montréal. Tabernacle! », Figaro magazine, 17 mai 1989.

ÉTATS-UNIS

Campbell, Bob. "Arcand takes skewed look at human identity", The Sunday StarLedger, May 28, 1995.

Car, Jay. "Remains reemerges with new life on film ", Boston Globe, June 16, 1995.

Cheshire, Godfrey. "Recline of Western Civ. ; Hands On; El Rampo", New York Press, May 31, 1995.

Denerstein, Robert. " "Hip" Canadian film empty at heart ", Rocky Mountain News, August 11, 1995.

Garcia, Marcia. "Jesus of Montreal", Film Reviews, ${ }^{\circ}$ 491-492.

Horton, Robert. "Some fun, fine romance in foreign films », The Herald, July 21, 1995.

John Iorn, "Filmmakers who work in a foreign language ", Philadelphia Inquirer, May 7, 1995.

James, Caryn. "Yearning to Connect, With a Killer Lurking", The New York Times, June 2, 1995.

Julian, Robert. "Filmmaker Denys Arcand Does It In English. Candy and Cadavers", Bay Area Reporter, June 22, 1995, p. 42-43.

Entre l'écho des voisins et celui des cousins. Les voix croisées de la réception critique... 
Kreps, Karen. "Jesus of Montreal", Box Office, vol. 126, n 7, July 1990, p. R-50.

LaSalle, Mick. "Loves Rules a Snapier Human Remains", SF Chronicle, June 23, 1995 , p. C3.

Neman, Daniel. "Love and Human Remains uninspired ", Times, August 1995.

O’Brien, Tom. "Jesus of Montrealn, Film Quarterly, vol. 44, n 1, 1990.

Rickey, Carrie. "Canadians in a contest of emotional detachment", Philadelphia Inquirer.

Verniere, James. "Love has haunting relevance", Boston Herald, June 16, 1995.

Whitty, Stephen. "Shifts in tone grate in Human Remains", San Jose Mercury News, June 23, 1995. 